inclusion. In this regard, policies and interventions require deep reflections on how best to balance opportunities and adversities, and sustain resilience to cope both with the present and future.

\title{
430 - Burnout and Negative Stereotypes: How They affect the Quality of Life of Professionals working in Nursing Homes.
}

\author{
López-Frutos, P., Pérez-Rojo, G., López, J., Noriega, C., Velasco, C., Carretero, M.I., Galarraga, L.
}

\section{Introduction}

The quality of life (QoL) of professionals working in nursing homes is often impaired by the stress that they experience daily. This sustained stress is known as burnout. A syndrome that ends up negatively affecting all areas of life and decreasing their quality of life. Negative stereotypes can alter how professionals perceive older adults. The literature has supported the relationship between high levels of burnout and negative stereotyping. These may moderate the impact of stress as a function of how they perceive stressors as reported by Lazarus and Folkman (1984).

\section{Objective}

This study aimed to analyze how burnout affects professionals' quality of life and how this relationship is moderated by their stereotypes towards the residents. The sample included 312 professionals working in nursing homes. Quality of life (QPL-35), burnout (MBI) and negative stereotypes towards older adults' health, motivation and personality (CENVE) were measured. To analyze the data, we performed linear regression models.

\section{Results:}

We found a negative relationship between burnout and quality of life ( $\left.p<0.001 ; R^{2}=0.47\right)$. The results of the moderation analysis show that they are significant regarding total stereotypes variable $(M 1: b=0.10$, $\mathrm{SE}=0.04, \mathrm{t}=2.18, \mathrm{p}=0.03)$, the health subscale $(\mathrm{M} 2: \mathrm{b}=0.14, \mathrm{SE}=0.04, \mathrm{t}=3.11, \mathrm{p}<0.001)$ and the socialmotivation subscale (M3: $b=0.09, S E=0.05, t=1.79, p=0.07)$. In contrast, character-personality subscale did not show any significant moderation ( $M 4: b=0.06, S E=0.05, t=1.25, p=0.21$ ).

\section{Conclusion:}

These results reflect the importance of investigating the role of stereotypes and their relationship with professionals' quality of life and burnout. Future intervention programs that aim to prevent negative stereotypes are needed not only to improve residents' quality of life, but also for professionals wellbeing and quality of life.

Funding: This research was funded by the Spanish Ministry of Economy and Competitiveness (grant no. PSI2016-79803-R).

\section{1 - Establishing a Canadian National ECHO Educational Program focused on Mental Health of Older Adults}

\author{
Authors: \\ David Conn, Lisa Sokoloff, Claire Checkland, Jasmeen Guraya, Vivian Ewa, Sid Feldman, Cindy Grief, \\ Andrea Hunter Navena Lingum, lan MacKay, Kiran Rabheru, Anna Santiago, Dallas Seitz, Devin Sodums, \\ Laurel Steed

\section{Background} \\ Project ECHO is a virtual, case-based capacity-building education program for healthcare providers. It \\ was developed in New Mexico, USA but, due to its effectiveness, the model has now spread to 40
}


countries around the globe. Baycrest, the Canadian Coalition for Seniors' Mental Health and the Canadian Academy of Geriatric Psychiatry collaborated to launch a national ECHO for mental health and aging. This partnership, coordinated by a cross-Canadian Steering Group, allows for broad reach, including registration of learning partners from almost all Canadian provinces and territories. The program was funded by the RBC Foundation.

\section{Methods}

ECHO COE: Mental Health pilot consisted of 2 cycles:

- 6 weekly sessions focused on broader mental health topics (e.g., delirium, mood disorders)

- 10 weeks with more specific topics (e.g., substance use disorders, sleep disorders)

Needs assessments of healthcare providers and older adults informed the program curricula. Evaluation included weekly satisfaction surveys, and pre and post evaluations.

\section{Results}

Participants:

- 154 healthcare providers participated in the 6-week session

- $39 \%$ of registrants were nurses or nurse practitioners, $35 \%$ allied health professionals, $14 \%$ physicians and $12 \%$ others

- 9 out of 10 provinces, 1 territory represented

Preliminary findings (based on the first 6 sessions):

- High overall satisfaction (average of 4.5 out of 5).

- $99 \%$ would recommend the program to others

- $67 \%$ had already shared information with team members and colleagues.

\section{Conclusion}

A national ECHO program is an effective way to bring together clinicians who work with and are interested in the mental health and wellbeing of older adults for education sessions, collaborative and mutual learning as well as for cross-jurisdictional knowledge transfer. Collaborative, cross-professional learning supports the exchange of best practice in mental health for older adults, supports the development of collegial national professional support and can address health system inequities. An international ECHO through IPA would be an exciting and valuable next step.

\section{2 - Ultrabrief Right Unilateral ECT in Older Adults with Catatonia: A Case Series and Literature Review}

Authors: Cristina Pritchett, MD.; Adriana Hermida, MD.; Amanda Tan B.S; Gregory Job, MD.

Objectives: Comparing Ultra-brief (UB) Right Unilateral Electroconvulsive Therapy (RU ECT) to the existing literature, this case series highlights the use of the treatment with geriatric patients.

\section{Methods:}

This is a retrospective chart review of 5 patients with catatonia who were successfully treated with UB RUL ECT. Medical records were reviewed for clinical data and ECT treatment. The existing literature is compared and contrasted with other published cases. Quick Inventory of Depressive Symptomatology (QIDS) was completed both pre-ECT and post-ECT. Clinical Global Impression-Improvement (CGI-I) scale was performed post-ECT. Response was defined as decrease of QIDS by $50 \%$ and remission was defined as QIDS $\leq 5$. CGI response was defined as $\mathrm{CGI}-\mathrm{I} \leq 2$.

\section{Results:}

All patients received UB RUL ECT treatment (mean age 67, 100\% female). $20 \%(n=2)$ were caucasian. $60 \%$ had mood disorders and $40 \%$ had psychotic disorders. Number of treatments in the acute 